the ability of cultured fibroblasts to esterify exogenously supplied cholesterol. This deficiency may be assayed in confirmation of the diagnosis when presentation is atypical. (Fink JK et al. Clinical spectrum of Niemann-Pick disease type C. Neurology August 1989; 39 : 1040-1049.

\begin{abstract}
COMENT. Mild intellectual impaiment presenting as poor school performance was the most camon initial neurologic abnormality. Additional presenting signs included ataxia, dysarthria, and impaired vertical gaze. Within three years of the initial deficit most of the patients had cognitive impaiment, abnormal vertical gaze and ataxia. Saccadic paresis was manifested by a camplaint of difficulty in reading or in descending stairs. Hepatosplenamegaly was first noted at varying ages from birth to 24 years with a mean age of six years. It preceded neurological abnormalities in one-half the patients and was found only in the early onset rapidly progressive group.
\end{abstract}

\title{
MITOCHONDRIAL ALTERATIONS IN RETT SYNDROME
}

Muscle biopsy findings in two patients with Ret $t$ syndrome are reported from the Departments of Obstetrics/Gynecology, Pediatrics, and Pathology, Medical College of Chio, Toledo, OH. Muscle biopsy was performed at 32 months of age and at 3 years 7 months of age. Light microscopy revealed fibers of uniform size with normal histochemistry. Electron microscopy revealed mitochondrial alterations including distention, vacuolation, and membranous changes. (Ruch A. Mi tochondrial alterations in Rett syndrome. Pediatr Neurol Sept/Oct 1989; $5: 320-3)$.

COMNENT. Abnormal mitochondria have been reported previously in the muscle biopsies of two patients with Rett syndrome (Eeg-Olofsson $O$ et al. Brain Dev 1988; 10:260). The findings

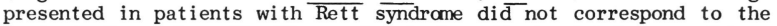
typical "ragged red" fibers found in mitochondrial myopathies. There are no biochemical or pathological findings specific to Rett syndrome but further studies of mitochondrial functioning in muscle may be warranted.

\section{INFANTILE MITOCHONDRIAL DISEASE}

A detailed clinical, pathologic, biochemical, and genetic analysis of a case of lethal infantile mitochondrial disease is reported from the Departments of Biochemistry, Pediatrics, Neurology and Nephrology, Emory University School of Medicine, Atlanta, GA. During the first three months of life the child showed increasing lethargy, hypotonia, difficulty in feeding and growth retardation. On admission at three months of age there was respiratory failure, bradycardia, hypotension, and severe lactic acidosis. Over the next 21 days the condition rapidly deteriorated with a progressive hypertrophic cardiamyopathy, hepatic dysfunction, and generalized seizure activity. The patient died with bradycardia and hypotension at four months of age. There were abnormalities in the striated muscles, smooth muscle, heart and liver but not in the central nervous system. Biochemical analysis revealed a cambined camplex I and IV 